\title{
Robust control method for bionic gait of machine legs based on time delay feedback
}

\author{
Lu Xing-Hua ${ }^{1, *}$, Huang Peng-Fen ${ }^{1}$, and Huang Wei-Peng ${ }^{1}$ \\ ${ }^{1}$ Huali College Guangdong University of Technology, 511325 Guangzhou Zengcheng, China
}

\begin{abstract}
The bionic machine leg is disturbed by the joint during the walking process, which is easy to produce time delay, which causes the robustness of the control of the machine leg is not good. In order to improve the robustness of the bionic gait control of the machine leg, a robust control method for the bionic gait of the machine leg based on time - delay feedback is proposed. The gait correlation parameters of robot leg are collected by sensor array, and the dynamic model of bionic gait is constructed. The fuzzy controller of bionic gait of robot leg is constructed by using time-delay coupling control method. The delayed feedback control error compensation method of machine leg correction is taken to improve the steady control performance of the robotic leg, reduce the steady-state error, improve the robustness of the control machine leg. The simulation results show that this method is robust to the bionic gait control of the machine leg. The output error of the gait parameter can quickly converge to zero, and the accurate estimation of the attitude parameter is stronger.
\end{abstract}

\section{INTRODUCTION}

As the booster device of human body, the robot leg has good application value in assisting the disabled to walk and rehabilitate exercise. The machine leg is arranged on the human body, the posture data acquisition is carried out by the sensing element, the control center of the machine leg is input according to the attitude parameter acquisition result. The legs of the machine are able to read the signals on the muscles, thereby controlling the machine leg switches and movements, mounting the legs of the machine legs in the legs of the disabled body, sending signals to the brain along the spinal cord. At this time, the mechanical leg through the electrode will be the muscle inside the signal collection, with the help of complex recognition software to process these signals, and achieve the power to operate in the design of the robot leg. The robust control of bionic gait of robot leg is the key to stable manipulation of robot leg. The research of bionic gait robust control method of robot leg has important application value in improving the stable control ability of robot leg[1].

The bionic machine leg is disturbed by the joint during the walking process, which is easy to produce time delay, which causes the robustness of the control of the machine leg is not good[2]. It is necessary to study robust control design for machine legs. Traditionally, the stability control method of the machine leg mainly includes fuzzy neural network control algorithm, PID control algorithm, slide - film control algorithm, steady state integral control algorithm and so on[3]. By constructing the corresponding controller, attitude error correction and disturbance compensation are carried out to realize the bionic gait control of the machine leg, and some research results are obtained. The reference [4] presents a single degree of freedom rate tracking and inertial machine leg gait control algorithm by unbalance compensation based on the robotic leg rate gyroscope step response by momentum moment feature inertia analysis, construct the steady-state equation realize gait stability of robotic leg control, improve the control precision, but the control algorithm the high computational cost of the machine, real-time gait leg state control is not good. In reference [5], an energy parameters self-tuning based on modified robotic leg gait fusion tracking control algorithm is proposed, the fuzzy adaptive PID is used for correction, robotic leg gait disturbance resistance is increased, but the algorithm requires a large number of machine leg attitude prior data and rules as a guide, when the lack of prior information, control effect is not good.

Aiming at the above problems, a robust control method for the bionic gait of the machine leg based on time - delay feedback is proposed. The gait correlation parameters of robot leg are collected by sensor array, and the dynamic model of bionic gait is constructed. The fuzzy controller of bionic gait of robot leg is constructed by using time-delay coupling control method. The delayed feedback control error compensation method of machine leg correction is taken to improve the steady control performance of the robotic leg, reduce the steady-state error, improve the robustness of the control machine leg. The simulation results show that this method is robust to the bionic gait control of the machine leg. Finally, the simulation experiment shows the superior performance of this method in improving the 
robustness control of the bionic gait of the machine leg.

\section{CONTROL PARAMETER ANALYSIS AND DYNAMIC MODEL OF MACHINE LEG CONTROL}

\subsection{Analysis of constraint parameters of machine leg control}

The gait correlation parameters of the robot leg are collected by sensor array, and the control constraint parameters are analyzed. The linear filtering and fitting of the gait posture data of the robot leg is carried out by multi-sensor fusion tracking under continuous time delay. In this paper, a linear time-varying system is constructed to fit the gait trajectory of the robot leg dynamically. It is assumed that the real robot leg is bionic with different attitude angles at a certain time $\boldsymbol{\beta}^{*}=\mathbf{V}(k)_{1} \boldsymbol{\Sigma}(k)_{1}^{-1} \mathbf{U}(k)_{1}^{T} \mathbf{Y}(k)$, wherein $\mathbf{V}(k)_{1} 、 \boldsymbol{\Sigma}(k)_{1}$ and $\mathbf{U}(k)_{1}$ are the results of SVD calculation for gait stability tracking control of machine legs, at the same time, a first order Taylor expansion approximation is $e^{-L_{m} s}=1-L_{m} s$, in the kernel space matrix of the system, the feedforward compensation design is introduced and the fuzzy PID controller is used to control the gait stability of the machine leg[6]. A time-delay coupled system is selected as the controlled object of the leg of the machine. When the transfer function is determined, $G_{m}(s)=G_{0}(s)$, $t_{m}=\tau$, the feedback signal of the gait tracking system of the robot leg is expressed as follows:

$$
H(s)+Y(s)=G_{m}(s) U(s)
$$

The gait control system of the robot legs forms a two degree of freedom IMC-PID controller. The multi-sensor information fusion method is used to measure the position and distribution of machine legs[7], and the attitude sensor transfer function is obtained:

$$
\left[\begin{array}{ll}
G_{11}(s) & G_{12}(s \\
G_{21}(s & G_{22}(s
\end{array}\right]=\left[\begin{array}{cc}
\frac{1.7 e^{-30 s}}{7 s+1} & \frac{0.59 e^{-27 s}}{8 s+1} \\
\frac{-0.6 e^{-25 s}}{10 s+1} & \frac{1.5 e^{-28 s}}{9 s+1}
\end{array}\right]
$$

In the upper form, the input vector of the controller is equivalent to the feedback signal drawn directly from the output of $G_{m}(s)$. When the system is affected by the interference vector $e^{-t_{m} s}$, gets the machine leg gait stability control loop transfer function and the system inertia meet transfer function:

$$
\frac{Y(s)}{R(s)}=\frac{G_{C}(s) G_{0}(s) e^{-\tau s}}{1+G_{C}(s) G_{0}(s)}
$$

The characteristic equation of the above transfer function has no delay term. The fourth order Runge-Kutta method is used to solve the delay error of the machine leg, and the error tracking compensation of the machine leg is carried out, and the delay link is obtained by Taylor approximation:

$$
Y(s)=\frac{e^{-L_{m} s}}{\left(\lambda_{1} s+1\right)} R(s)+\frac{\left(\lambda_{2} s+L_{m}\right) s}{\left(\lambda_{2} s+1\right)} D(s)
$$

When the parametric model of the gait control of the machine leg is inaccurate, there is a characteristic equation:

$$
\frac{Y(s)}{R(s)}=\frac{G_{C}(s) G_{0}(s) e^{-\tau s}}{1+G_{C}(s) G_{m}(s)+G_{C}(s)\left(G_{0}(s) e^{-\tau s}-G_{m}(s) e^{-t_{m} s}\right)}
$$

Power gain in the process of time delay control is $K=\Delta K \cdot K_{m}$, wherein $\Delta K>0$, The characteristic equation is still influenced by the time - delay link, the speed change process of the gait of the machine leg can be expressed as a continuous progressive state variable, and the progressive variable of the gait posture of the machine leg is evaluated to obtain the following non smooth time varying function of the machine leg gait track tracking control system:

$$
\begin{aligned}
& \dot{x}(t)=A x(t)+B x\left(t-d_{1}(t)-d_{2}(t)\right) \\
& x(t)=\phi(t) \quad t \in[-h, 0]
\end{aligned}
$$

According to optimization conditions for linearized small perturbations, when the disturbance vector error matrix $x(t)=\left[x_{1}(t), x_{2}(t), \ldots, x_{t}(t)\right]^{T}$ is converged, the control constraint parameters of the machine legs have self- correlation[8].

\subsection{Kinetic model of motion of machine leg}

On the basis of the above analysis of control constraint parameters, a mathematical model for longitudinal motion of machine legs is built. The motion equations of machine legs mainly include centroid motion equation, centroid dynamic equation, and the coherent distribution source model of machine legs in relative inertia space:

$$
\mathbf{z}(\mathbf{t})=\sum_{i=1}^{p} s_{i}(t) \mathbf{b}_{\mathbf{i}}\left(\theta_{i}\right)+\mathbf{n}(t)
$$

Wherein:

$$
\mathbf{b}_{\mathbf{i}}\left(\theta_{i}\right)=\int_{-\pi}^{\pi} \mathbf{a}(\boldsymbol{\theta}) g_{i}\left(\theta-\theta_{i}\right) d \theta
$$

The covariance matrix of the gait data vector $\mathbf{z}(\mathbf{t})$ of the machine leg observed by the attitude sensor can be expressed as:

$$
\begin{gathered}
\mathbf{R}=E\left[\mathbf{z}(\mathbf{t}) \mathbf{z}^{\mathbf{H}}(\mathbf{t})\right]=\mathbf{B} \mathbf{P}_{\mathbf{s}} \mathbf{B}^{\mathbf{H}}+\sigma_{n}^{2} \mathbf{I}_{\mathbf{M}} \\
\mathbf{B}=\left[b_{1}\left(\theta_{1}\right), b_{2}\left(\theta_{2}\right), \ldots, b_{q}\left(\theta_{q}\right)\right]^{T}
\end{gathered}
$$

Wherein $\mathbf{B}=\left[b_{1}\left(\theta_{1}\right), b_{2}\left(\theta_{2}\right), \ldots, b_{q}\left(\theta_{q}\right)\right]^{T}$, the center direction of arrival of the gait attitude of the robot leg is $\theta_{i}$ and the extended angle $\sigma_{i}$. The singular value of the external torque covariance matrix acting on the robot leg gait inertial navigation gyroscope is decomposed into:

$$
\mathbf{R}=\mathbf{U}_{\mathrm{s}} \boldsymbol{\Lambda}_{\mathrm{s}} \mathbf{U}_{\mathrm{s}}^{\mathrm{H}}+\mathbf{U}_{\mathrm{n}} \boldsymbol{\Lambda}_{\mathrm{n}} \mathbf{U}_{\mathrm{n}}^{\mathrm{H}}
$$

The column vectors of damping moment matrix $\mathbf{U}_{\mathrm{s}}$ and $\mathbf{U}_{\mathbf{n}}$ generated by dampers are derived from singular values, respectively. $\sigma_{1}, \sigma_{2}, \ldots \sigma_{q}$ and $\sigma_{n}$ are singular vectors correspondingly, under the influence of 
the interference moment, the kinematic output state equation of the machine leg can be described as:

$$
\left\{\begin{array}{l}
x(k+1)=A x(k)+B K x\left(k-\tau_{k}\right) \\
z(k)=C x(k)+D K x\left(k-\tau_{k}\right)
\end{array}\right.
$$

According to the above analysis, the dynamic equation of the robot leg is constructed, which provides the accurate data input basis for the bionic gait robust control of the robot leg[9].

\section{CONTROL METHOD OPTIMIZATION DESIGN}

\subsection{Feedback compensation control with time - delay}

On the basis of using sensor array to collect gait correlation parameters of machine leg and constructing dynamic model of bionic gait, the optimal design of control algorithm is carried out. In this paper, a robust control method for bionic gait of robot legs based on time-delay feedback is proposed[10].

In the case of continuous disturbance and input constraint, the gait tracking sequence of the input machine leg is obtained as $u(n)$, state stability control output is $x(n)$, thus, a two-degree-of-freedom control active disturbance rejection data tracker with time-delay is constructed. The relationship between input and output is as follows. :

$$
x(n)+\sum_{k=1}^{p} a_{i} x(n-k)=\sum_{r=0}^{m} b_{r} u(n-r)
$$

Assume $\Delta x_{k}=\alpha p_{k}$, and $\alpha$ is error feedback correction coefficient. The fuzzy controller of bionic gait state of the robot leg is constructed by using the time-delay coupling control method. The original data collected by the gyroscope are processed by Kalman filter, and the output parameter sequence is obtained as follows: $x_{1}=y, x_{2}=\dot{y}, \cdots, x_{n}=y^{(n-1)}$, then the above control equation of state can be rewritten:

$$
\left\{\begin{aligned}
\dot{x}_{1}= & x_{2} \\
& \vdots \\
\dot{x}_{n}= & f(X, t)+\Delta f(X, t)+d(t)+b(X, t) u
\end{aligned}\right.
$$

Wherein, $X=\left[x_{1}^{T}, x_{2}^{T} \cdots x_{n}^{T}\right]^{T}=\left[x_{1}^{T}, \dot{x}_{1}^{T} \cdots x_{1}^{(n-1) T}\right]^{T}$, the adaptive law is designed. The attitude angle of the gyroscope is obtained by the four elements method, and the state of the gait correction system of the machine leg is obtained. $X=\left[x_{1}^{T}, x_{2}^{T} \cdots x_{n}^{T}\right]^{T}=\left[x_{1}^{T}, \dot{x}_{1}^{T} \cdots x_{1}^{(n-1) T}\right]^{T}$. Adaptive equalization is realized in a limited time. According to the control rules of bionic gait stability of the robot leg, the current position of the robot leg is calculated based on the standard energy gradient correction of the Terminal sliding mode surface, get :

$$
\begin{aligned}
u(t)= & -b(x, t)^{-1}\left[f(X, t)-x_{1 d}^{(n)}-p(t)^{(n)}+C_{n}^{-1} \sum_{k=1}^{n-1} C_{k}\left(e^{(k)}-p(t)^{(k)}\right)\right] \\
& -b(x, t)^{-1} \frac{C_{n}^{T} \sigma}{\left\|C_{n}^{T} \sigma\right\|}[F(X, t)+D(t)+K]
\end{aligned}
$$

Wherein, $K$ is positive integer, when the machine leg misses the optimal energy gradient correction segment.

\section{Assumption 1 :}

$p_{i}(t): R_{+} \rightarrow R, p_{i}(t) \in C^{n}[0, \infty), \dot{p}_{i}, \cdots, p_{i}^{(n)} \in L^{\infty}$. For a walking state, the time parameter $T>0$, parameter estimation of tracking error $p_{i}(t), \dot{p}_{i}(t), \cdots, p_{i}(t)^{(n)}$ are $0, \quad i=1,2, \cdots, m$. $C^{n}[0, \infty)$ expressed in $[0, \infty)$, select function $p_{i}(t)$ is:

$$
p_{i}(t)= \begin{cases}\sum_{k=0}^{n} \frac{1}{k !} e_{i}(0)^{(k)} t^{k}+\sum_{j=0}^{n}\left(\sum_{l=0}^{n} \frac{a_{i j}}{\left.T^{j-l+n+1} e_{i}(0)^{(l)}\right) \cdot t^{j+n+1}}\right. & 0 \leq t \leq T \\ 0 & t>T\end{cases}
$$

Under the control of longitudinal motion, $a_{i j}$ is terminal sliding mode surface of the system, it can be determined through the adaptive registration of the error correction controller, through the analysis and design of the system state, with the cumulative time varying method will be extended to the whole stability of the solution of horizontal plane and vertical plane machine leg gait analysis, the static and dynamic characteristics of the machine leg, the leg when the machine is perturbed, the time delay compensation, improve the real-time control of the robotic leg.

\subsection{Machine leg gait control error correction}

Ensure the global robustness and stability of the closed loop system of machine legs. The time delay feedback compensation and error correction are used, and the weight matrix obtained by layer inertial fusion tracking method is adopted as $w^{k}=\left(w_{1}^{k}, w_{2}^{k}, \cdots, w_{m}^{k}\right)^{\top}$ and perturbation matrix $\varsigma=\left(s_{i j}\right)_{m \times n}, \quad$ comprehensive evaluation degree matrix $E$, the formula is expressed as follows:

$$
\begin{aligned}
& E=w^{k} \cdot \varsigma=\left(e_{1}, e_{2}, \cdots, e_{n}\right) \\
& =\left(w_{1}, w_{2}, \cdots, w_{m}\right) \cdot\left[\begin{array}{ccc}
s_{11} & \cdots & s_{1 n} \\
\vdots & \ddots & \vdots \\
s_{m 1} & \cdots & s_{m n}
\end{array}\right]
\end{aligned}
$$$$
e_{j}=\sum_{k=1}^{m} w_{k} \cdot s_{k j}
$$

wherein, $\quad e_{j}=\sum_{k=1} w_{k} \cdot s_{k j} . e_{j}$ is comprehensive evaluation of time delay error of machine legs, according to the degree of the relative merits of each feature size can be evaluated.

The state function of the bionic gait robust control of the machine legs is:

$$
F_{i}=\left(f_{1}, f_{2}, \cdots, f_{m}\right)
$$

Indicator Set is

$$
X_{j}=\left(x_{1}, x_{2}, \cdots, x_{n}\right)
$$

The inertia weight of bionic gait control of robot leg is obtained:

$$
D=\left[\begin{array}{cccc}
d_{11} & d_{12} & \cdots & d_{1 n} \\
d_{21} & d_{22} & \cdots & d_{2 n} \\
\vdots & \vdots & \ddots & \vdots \\
d_{m 1} & d_{m 2} & \cdots & d_{m n}
\end{array}\right]
$$

Set the control target scheme is $\vartheta, \vartheta=\left(\theta_{1}, \theta_{2}, \cdots, \theta_{n}\right)$, the optimal solution of the positioning angle of the robot 
leg is:

$$
\theta_{i}=\left\{\begin{array}{c}
\max d_{i j}, \quad \text { index is the benefit index } \\
\min d_{i j}, \text { indicator is cost based indicators } \\
\text { Appro, index is a moderate index }
\end{array}\right.
$$

The time delay feedback compensation method is used to correct the control error of the machine leg, and the optimal step length of the machine leg is obtained:

$$
R_{p}=R_{\text {TURN }}+\frac{R_{H A C} \psi_{H}}{\cos 30^{\circ}}-X_{H A C}
$$

Wherein:

$$
R_{\text {TURN }}=\frac{R_{T A C}\left|\Delta \psi_{H A C}\right|}{\cos \phi}+\sqrt{A^{2}+B^{2}} \quad R_{T A C}=\frac{v_{2} \cos \gamma}{g \tan \phi}
$$

In summary, the robust control of the bionic leg gait machine, improve the stability and robustness of the machine walking legs.

\section{SIMULATION EXPERIMENT AND RESULT ANALYSIS}

In order to test the application performance of the method in the robustness control of the robot leg gait, the simulation experiment is carried out, and the algorithm design of the simulation experiment runs on the Matlab7.0 numerical simulation platform. The hardware environment of the experiment is: The attitude information fusion matrix of the machine leg is:

$$
\varsigma=\left(s_{i j}\right)_{m \times n}=\left[\begin{array}{cc}
0.6453 & 1 \\
1 & 0.4 \\
1 & 0.9982 \\
1 & 0.7385 \\
1 & 0.0546 \\
0.9918 & 1 \\
1 & 1
\end{array}\right]
$$

The expansion angles of the data sampling array sources for the input of the gait attitude data are $\Delta_{1}=5^{0}, \Delta_{2}=8^{0}$ respectively, the signal-to-noise ratio (SNR) of gait disturbance of robot leg is $2 \mathrm{~dB}$, main sidelobe height ratio is $30 \mathrm{~dB}$, weight matrix derived from analytic hierarchy process is $w^{k}$ and same time matrix ${ }^{\varsigma}$, comprehensive evaluation degree matrix of computational features $E$ :

$$
\begin{aligned}
& E=w^{k} \cdot \varsigma=\left(e_{1}, e_{2}, \cdots, e_{n}\right) \\
& =\left(w_{1}, w_{2}, \cdots, w_{m}\right) \cdot\left[\begin{array}{ccc}
s_{11} & \cdots & s_{1 n} \\
\vdots & \ddots & \vdots \\
s_{m 1} & \cdots & s_{m n}
\end{array}\right] \\
& =\left(\begin{array}{ll}
0.9780 & 0.9102
\end{array}\right)
\end{aligned}
$$

The decision matrix of bionic gait stability control is constructed as follows:

$$
D=\left[\begin{array}{cc}
2.6366 & 1.7014 \\
2 & 5 \\
0.9517 & 0.9500 \\
6429.3329 & 4748.0429 \\
48.76878 & 893.90164 \\
0.2913 & 0.2889 \\
5 & 5
\end{array}\right]
$$

Thus, the optimal solution of gait parameters of the machine legs is obtained:

$\vartheta=(1.7014,2,0.9517,6429.3329,48.76878,0.2889,5)$

The bionic gait control simulation experiment of the machine leg is carried out according to the result of the calculation of the parameters mentioned above, the results of the gait parameter tracking control of the machine leg are shown in Figure 1.

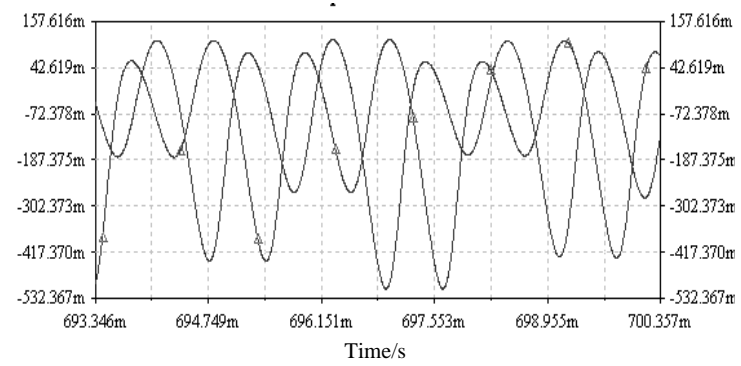

Fig. 1 Gait parameter tracking control results for machine legs

Figure 1 analysis shows that this method of robotic leg gait tracking control, steady attitude parameter of the machine leg tracking control performance is better, in order to carry out the error convergence of bionic robotic leg gait control, quantitative analysis method in this paper, the test machine leg control parameters of the desired output and the actual output, control performance curve is shown in Figure 2.

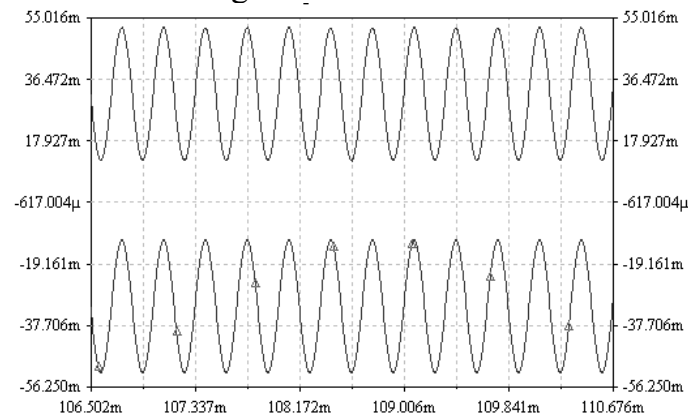

Fig. 2 Bionic gait control performance curve of machine leg

Figure 2 shows that the control convergence of this method is good. The expected output is in good agreement with the actual output. Table 1 gives the error of using different methods to control the machine leg. The analysis shows that the error of the method in this paper is lower. The robustness of the robot leg control is guaranteed.

Table 1 Mean absolute error of two algorithms

\begin{tabular}{cccc}
\hline \multicolumn{2}{c}{ Algorithms } & This method & PID \\
\hline \multirow{2}{*}{$\begin{array}{c}\text { Absolute } \\
\text { error }\end{array}$} & $\begin{array}{c}\text { position } \\
(\mathrm{m})\end{array}$ & 1.021 & 3.831 \\
\cline { 2 - 4 } & $\begin{array}{c}\text { velocity } \\
(\mathrm{m} / \mathrm{s})\end{array}$ & 0.212 & 1.092 \\
\hline
\end{tabular}

\section{CONCLUSION}

In order to improve the robustness of the bionic gait control of the machine leg, a robust control method for the bionic gait of the machine leg based on time - delay feedback is proposed. The gait correlation parameters of robot leg are collected by sensor array, and the dynamic 
model of bionic gait is constructed. The fuzzy controller of bionic gait of robot leg is constructed by using time-delay coupling control method. The delayed feedback control error compensation method of machine leg correction is taken to improve the steady control performance of the robotic leg, reduce the steady-state error, improve the robustness of the control machine leg. The simulation results show that this method is robust to the bionic gait control of the machine leg. The output error of the gait parameter can quickly converge to zero, and the accurate estimation of the attitude parameter is stronger, it has good application value in bionic gait control.

\section{ACKNOWLEDGMENTS}

This project is supported by and 2017 Undergraduate Scientific and Technological Innovation Project Fund of Guangdong Province (pdjh2017a0938).

\section{REFERENCES}

1. Lee $\mathrm{W}$, Bang $\mathrm{H}$, Leeghim H. Cooperative localization between small UAVs using a combination of heterogeneous sensors $[\mathrm{J}]$. Aerospace Science and Technology, 2013, 27(1): 105-111.

2. GE Lizhi. Visual simulation of UUV Attack Model Based on Whole Trajectory Control Analysis[J].Ship Electrolic Engneering,2015,35(3):137-141.

3. WANG Xun, ZHANG Daibing, SHEN Lincheng. A Virtual Force Based Path Following Approach for Unmanned Aerial Vehicles. ROBOT, 2016, 38(3): 329-336.
4. BROX T and MALIK J. Large displacement optical flow: descriptor matching in variational motion estimation[J]. IEEE Transactions on Pattern Analysis and Machine Intelligence, 2010, 33(3): 500-513. doi: 10.1109/TPAMI.2010. 143.

5. EVANGELIO R H, PATZOLD M, and KELLER I. Adaptively splitted GMM with feedback improvement for the task of background subtraction[J]. IEEE Transactions on Information Forensics and Security, 2014, 9(5): 863-874.

6. HE Da-kuo GAO Fei-xue YANG Le WU Wei. Improved adaptive quasi-sliding mode decoupling control for a class of unknown MIMO nonlinear discrete-time systems. Control and Decision, 2016, 31(05): 783-789.

7. HUANG Guo-shun WEN Han. Uncertainty measures of rough sets based on boundary region and knowledge granularity. Control and Decision, 2016, 31(06): 983-989.

8. DOU Huijing, WANG Qianlong, ZHANG Xue. A Joint Estimation Algorithm of TDOA and FDOA Based on Wavelet Threshold De-noising and Conjugate Fuzzy Function. JEIT, 2016, 38(5): 1123-1128.

9. Li Xu, Peng Huan, Wang Chunhui. APD optimal gain control investigation for spaceborne laser range finder. Infrared and Laser Engineering, 2016, 45(5): 520001-0520001(5).

10. DU Xuedan, CAI Yinghao, LU Tao, WANG Shuo, YAN Zhe. A Robotic Grasping Method Based on Deep Learning. ROBOT, 2017, 39(6): 820-828,837. 